\title{
A Flexible Inductive Coil Tag for Household Two-Wire Current Sensing Applications
}

\author{
Yung-Chang Chen, Sung-Chu Yu, Shih-Hsien Cheng, and Yu-Ting Cheng, Senior Member, IEEE
}

\begin{abstract}
This paper presents a flexible inductive coil tag for sensing the electric current in the two-wire power cords of household goods. The tag is fabricated using a complementary metal-oxide semiconductor compatible SU-8 flexible technology which provides unique device characteristics of low-cost, reliability and pervasiveness. With a 30-turns coil design in an area of $0.5 \times 1 \mathrm{~cm}^{2}$, the coil tag can provide sensitivity of 18 and $21 \mu \mathrm{V} / \mathrm{A}$ for detecting 50- and $60-\mathrm{Hz}$ electric current in the ampere regime, respectively.
\end{abstract}

Index Terms-Current sensor, demand-response electricity monitoring systems, flexible, proximity.

\section{INTRODUCTION}

$\mathbf{I}$ MPROVING energy efficiency has become a worldwide government policy in determining a national technology development roadmap owing to natural resource deficiency and global climate warming. Deployment of demand-response (DR) electricity monitoring systems to manage electricity usage in residential areas is a cost-effective way to accomplish this goal [1]. Electric current sensor is one of the key components in the system and must be designed with the characteristics of low-cost, pervasive and easy-implementation for household-goods power monitoring system applications.

Previously, various current sensors including current transformer, Rogowski coil, Hall effect, fluxgate, AMR, piezoMEMS and so on have been developed and reviewed [2]-[12]. These sensors are designed to detect the magnetic field generated by the current source flowing through the power line. For the application of power monitoring in household appliances whose electrical cable is a two-wire power line like AWG (American Wire Gauge) wires in general, the sensor types of the current transformer, Rogowski coil [6], [7], Hall effect sensor [8], [9], and fluxgate [10]...etc are not practical in use. While the sensors are used for the current detection, either the

Manuscript received December 5, 2011; accepted January 1, 2012. Date of publication January 9, 2012; date of current version April 26, 2012. This work was supported by ITRI under the Grant $99 \mathrm{C} 117$ project, in part by NSC under the Grant 100-2220-E-009-007 project and the Ministry of Education in Taiwan under ATU Program. The associate editor coordinating the review of this paper and approving it for publication was Dr. Patrick Ruther.

Y.-C. Chen, S.-C. Yu, and Y.-T. Cheng are with the Department of Electronics Engineering and the Institute of Electronics, National Chiao Tung University, Hsinchu 30010, Taiwan (e-mail: ycchen.ee97g@g2.nctu.edu.tw; h2oduoljr@gmail.com; ytcheng@mail.nctu.edu.tw).

S.-H. Cheng is with the Institute of Electrical Control Engineering, National Chiao Tung University, Hsinchu 30010, Taiwan, and the Green Energy and Environment Research Laboratories, Industrial Technology Research Institute, Chutung 31040, Taiwan (e-mail: shrshianjeng@itri.org.tw).

Color versions of one or more of the figures in this paper are available online at http://ieeexplore.ieee.org.

Digital Object Identifier 10.1109/JSEN.2012.2183587

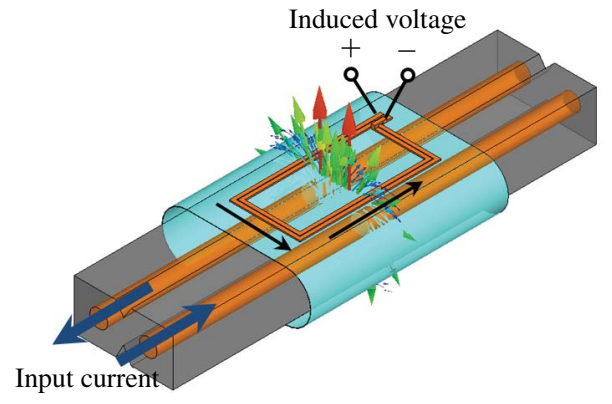

Fig. 1. Scheme of the proposed inductive coil tag for current sensing.

fire or ground wire must be separated from the other one in the two-wire cable and only one of the wires is then encircled by the sensors. Therefore, a non-intrusive current detection scheme will be the most acceptable solution which just simply employs the sensors to the nearby magnetic field generated by the current for the detection and related sensor technology must be developed.

Recently, a new piezoelectric MEMS-based current sensor has been developed for detecting the current information without breaking the wires [11], [12]. The sensor was devised with a piezoelectric cantilever beam where a permanent magnet was deposited on the top of its free end, so the strength of the central magnetic field on the top of the power cable could be detected from the actuated beam by magnetic force. In this work, another cost-effective, flexible, inductive coil tag closely fitted with the power cords of household appliances as shown in Fig. 1 is, therefore, developed for non-intrusive current detection. Based on Faraday's law of induction, the proposed sensor tag devised with an inductive coil for sensing the same central magnetic field also provides another sensor technology for advancing current sensing module technique for widespread use.

\section{DESIGn AND FABRiCATION}

Fig. 2 shows the geometry of a $1.25 \mathrm{~mm}^{2} / 2 \mathrm{C}$ power cord (Fig. 2a) and the simulation result of the magnetic flux intensity distribution on the cord with a $1 \mathrm{~A}, 60 \mathrm{~Hz}$ current input, respectively (Fig. 2b). According to Faraday's law of induction, the induced voltage on an open-loop conductive coil is proportional to the rate of the magnetic flux change through the coil and it can be calculated as follows [13]:

$$
V_{\text {in }}=-\frac{d \Phi}{d t}=-N \cdot A \cdot \frac{d B}{d t}
$$




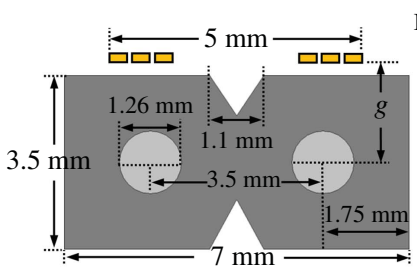

(a)

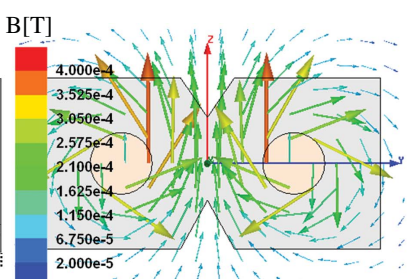

(b)
Fig. 2. (a) Geometry of $1.25-\mathrm{mm}^{2} / 2 \mathrm{C}$ power cord on top of which a sensing coil is put for the current sensing. (b) Simulation of magnetic flux distribution on $2 \mathrm{C}$ power cord at $1 \mathrm{~A}, 60-\mathrm{Hz}$ input current.

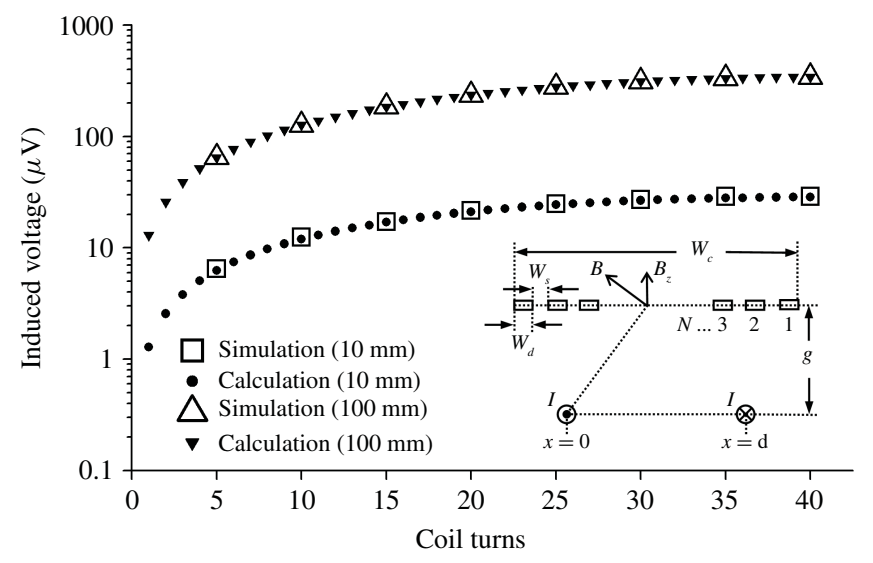

Fig. 3. Simulation (10 and $100 \mathrm{~mm})$ and calculation $(10$ and $100 \mathrm{~mm})$ of induced voltage versus coil turns. The inset shows the structural parameters of theoretical model.

where $\Phi, A$, and $N$ are the magnetic flux passing through the coil, the area and turns of the coil, respectively. The simulation indicates the location right on the top central area of the power cord (Fig. 2a) can make a coil for current sensing with the highest open-loop induced voltage resulted by the existence of the largest vertical magnetic flux intensity change. Meanwhile, the flux intensity would decrease with the separation between the coil and this cord. The sensing coil must, therefore, be designed with better proximity to the power cord for maximizing its induced voltage output. In order to accomplish the criteria, a flexible inductive coil tag is fabricated for current sensing application using a previously developed SU-8 flexible technology [14].

Fig. 3 shows the induced voltage versus the coil turns of the typical planar inductive coil with the geometry design and spatial relation to the sensed two-wire power cord as shown in the inset figure with a cross sectional view. In such a typical planar coil design, the induced voltage contributed by each turn of the coil can be further derived as follows from Eq. (1):

$$
\begin{aligned}
\Phi_{n} & =\int \vec{B} \cdot d \vec{A}=\frac{\mu_{0} I \cos \omega t}{\pi} c_{n} \int_{a_{n}}^{b_{n}} \frac{x}{x^{2}+g^{2}} d x \\
V_{i n} & =-\sum_{n=1}^{N} \frac{d \Phi_{n}}{d t}=\frac{\omega \mu_{0} I \sin \omega t}{2 \pi} \sum_{n=1}^{N} c_{n} \ln \left(\frac{b_{n}^{2}+g^{2}}{a_{n}^{2}+g^{2}}\right) \\
a_{n} & =\frac{d}{2}-\frac{1}{2}\left[w_{c}-2 n \cdot w_{d}-2(n-1) \cdot w_{s}\right] \\
b_{n} & =\frac{d}{2}+\frac{1}{2}\left[w_{c}-2 n \cdot w_{d}-2(n-1) \cdot w_{s}\right] \\
c_{n} & =L-2 n \cdot w_{d}-2(n-1) \cdot w_{s}
\end{aligned}
$$

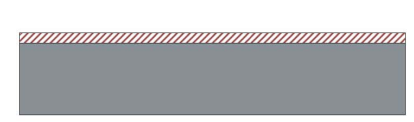

(a)

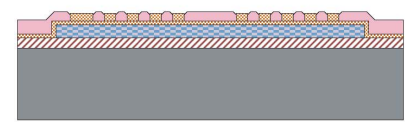

(c)

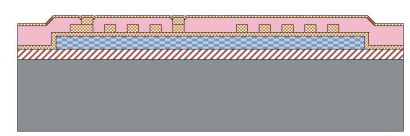

(e)

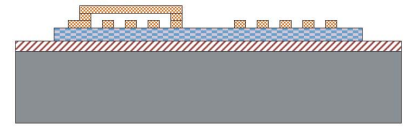

(g)

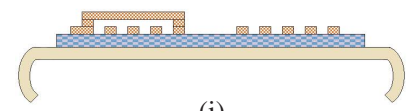

(i)

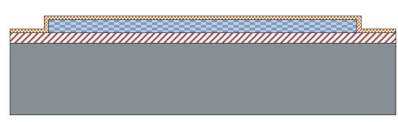

(b)

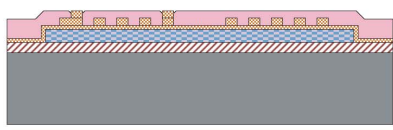

(d)

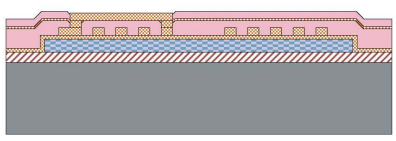

(f)

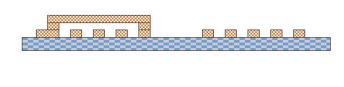

(h)

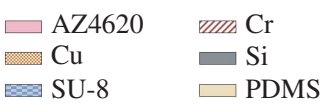

Fig. 4. Fabrication process flow of the flexible current sensor tag: (a) sacrificial $\mathrm{Cr}$ layer deposition, (b) SU-8 photoresist mold with a $\mathrm{Ti}(30 \mathrm{~nm}) / \mathrm{Cu}(150 \mathrm{~nm})$ adhesion/seed layer, (c) 5 - $\mu \mathrm{m}$-thick electroplating $\mathrm{Cu}$, (d) coil via plating, (e) another $\mathrm{Cu}$ seed layer deposition, (f) $5-\mu$ m-thick $\mathrm{Cu}$ coil bridge fabrication, (g) removal of photoresist, seed layer, and adhesion layer, (h) flexible coil tag release, and (i) 26- $\mu$ m-thick PDMS substrate attachment to form the current sensing tag.

where $\Phi_{n}$ is the $n_{t h}$ magnetic flux passing through the $n_{t h}$ turn of the coil, $\omega$ is the angular frequency, $N$ is total turns of the coil, $w_{d}$ is the metal line width, $w_{s}$ is the line spacing between each turn, $w_{c}$ is total sensor width, $L$ is total sensor length, $d$ is the distance between $2 \mathrm{C}$ power central points, and $g$ is the gap distance between coil and the power cord. Fig. 3 shows a good data match between Ansoft Maxwell simulation for both cases of $\mathrm{L}=10$ and $100 \mathrm{~mm}$ and the model derivation in terms of the induced voltage versus coil turns of the sensor detecting a $1 \mathrm{~A}, 60 \mathrm{~Hz}$ current input in a $2 \mathrm{C}$ power cord. For the coil with an area of $0.5 \times 1 \mathrm{~cm}^{2}, 30 \mu \mathrm{m}$ in line width, $30 \mu \mathrm{m}$ in line-spacing and a distance of $56 \mu \mathrm{m}$ to the cord top, the induced voltage is $26.6 \mu \mathrm{V}$ and becomes saturated with more turns added while the coil turns become 30 . The distance between the coil tag and the top surface of the cord is limited by the fabrication process where the flexible tag thickness is SU-8(30 $\mu \mathrm{m}) / \mathrm{PDMS}(26 \mu \mathrm{m})$ which will be introduced later. The match verifies the accuracy of the analytical model and shows that the induced voltage can be further enlarged with the coil size, i.e. $0.5 \times 10 \mathrm{~cm}^{2}$. By lengthening the coil from 10 to $100 \mathrm{~mm}$, we can have an order of magnitude enhancement in the induced voltage.

The flexible inductive coil tag is fabricated using a threelayer $\mathrm{Cu}$ electroplating process with the wafer level SU-8 flexible technology [14]. The fabrication begins with $30 \mathrm{~nm}$-thick sacrificial Cr layer deposition on a silicon substrate for the tag release (Fig. 4a). A $30 \mu \mathrm{m}$-thick SU-8 photoresist is then spincoated, patterned, and deposited with a $\mathrm{Ti}(30 \mathrm{~nm}) / \mathrm{Cu}(150 \mathrm{~nm})$ adhesion/seed layer (Fig. 4b). After that, AZ4620 photoresist is patterned on the adhesion/seed layer and used as a mold for a $5 \mu \mathrm{m}$-thick electroplating $\mathrm{Cu}$ (Fig. 4c) which is followed by another photoresist patterning and electroplating process 


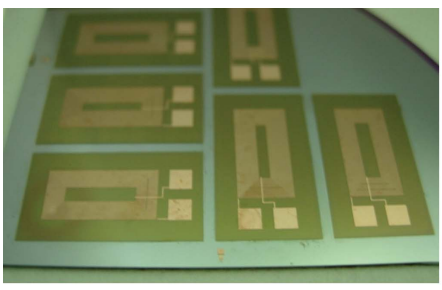

(a)

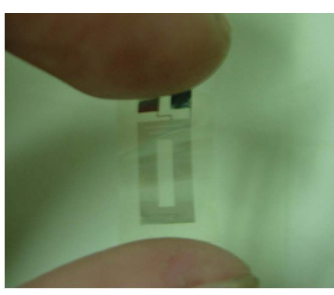

(b)
Fig. 5. Optical photographs of the flexible inductive coil tag fabrication (a) before sacrificial $\mathrm{Cr}$ layer release and (b) release from the substrate.

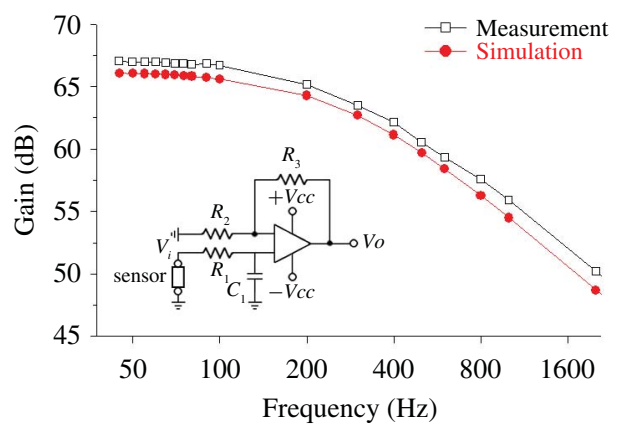

Fig. 6. Transfer function whose circuit gain is $66 \mathrm{~dB}$ in SPICE simulation and $66.9 \mathrm{~dB}$ in measurement. Lower left: active low-pass filter circuit scheme.

steps to form a coil via (Fig. 4d). Once the via plating is done, another $\mathrm{Cu}$ seed layer is deposited (Fig. 4e), coated by patterned AZ4620 photoresist and electroplated with a $5 \mu$ m-thick $\mathrm{Cu}$ for the coil bridge fabrication (Fig. 4f). The substrate is then sequentially dipped in acetone, $\mathrm{Cu}$ etchant, and BOE solution to remove the photoresist, seed layer, and adhesion layer, respectively (Fig. 4g). At final, the flexible coil is released from the silicon substrate by dipping in a $\mathrm{Cr}$ etchant $\left(\mathrm{H}_{2} \mathrm{O}: \mathrm{HCl}=100: 20\right)$ for the sacrificial layer removal (Fig. 4h) and attached to a $26 \mu \mathrm{m}$ thick PDMS substrate to form the current sensing tag where the substrate can be bended to closely fit with the power cords (Fig. 4i). Fig. 5 shows the optical photographs of the as-fabricated coil tag before (Fig. 5a) and after (Fig. 5b) sacrificial $\mathrm{Cr}$ layer release, respectively.

\section{RESUlts AND Discussion}

According to the aforementioned analysis, the sensed signal is about several tens of $\mu \mathrm{V} / \mathrm{A}$ which requires a signal amplifier for the electrical characterization of the flexible coil. Fig. 6 shows an active low-pass filter circuit is designed with $66.9 \mathrm{~dB}$ gain which is controlled by the resistance ratio of $R_{1}\left(R_{2}\right)$ to $\mathrm{R}_{3}$. In the work, 300 and $1 \mathrm{M} \Omega$ variable resistors are chosen for a flat gain response in a frequency range of 45 to $100 \mathrm{~Hz}$ which is a typical range of the household AC power signals in the world. The low noise operational amplifier, OP27G (Texas Instruments Inc.), is used for the gain stage. The flexible inductive coil tag is then soldered with two metal wires connected to the circuit formed on a PCB.

Fig. 7 shows the setup for current sensing. All feedthroughs are ground-shielded to avoid electrical signal coupling and interference with the testing system. The senor tag shown in

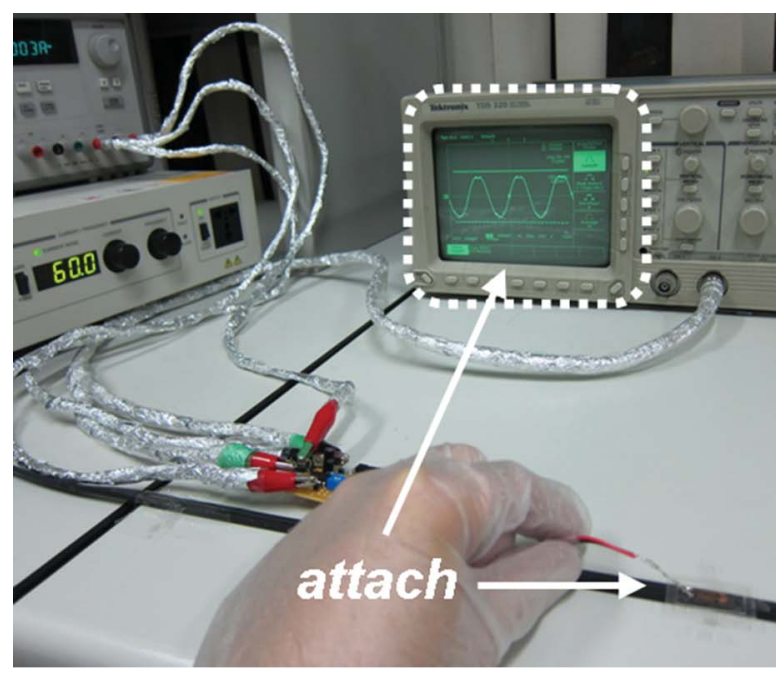

(a)

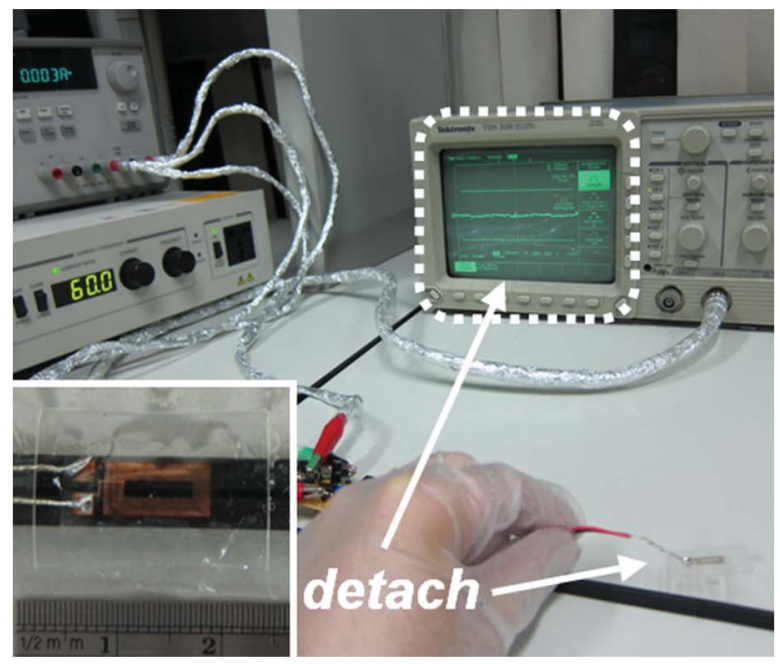

(b)

Fig. 7. Oscilloscope monitor: (a) sinusoidal wave while the sensor pastes on and (b) no signal output while the sensor takes up from the power cord with $3 \mathrm{~A}, 60 \mathrm{~Hz}$ current input. The inset of (b) shows the as-fabricated device.

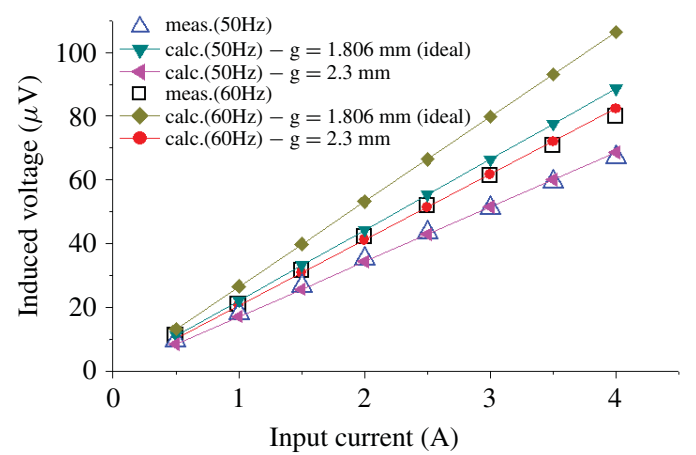

Fig. 8. Measured and calculated voltage induced in the coil versus the input current in the cord. The data have been converted into the original signals without amplification.

the inset of (b) is attached to (Fig. 7a)/detached from (Fig. 7b) a $2 \mathrm{C}$ power cord loaded with the current source (ELGAR CW801M) with a $1 \mathrm{~A}, 60 \mathrm{~Hz}$ current output. The disappearance of the sensed signal in the oscilloscope, while the tag is detached, validates the sensing mechanism of magnetic flux 
TABLE I

List OF CURRENT SENSORS

\begin{tabular}{ccccc}
\hline \hline & $\begin{array}{c}\text { Pernia } \text { et al. } \\
(\mathbf{1 9 9 8})[\mathbf{1 9 ]}\end{array}$ & $\begin{array}{c}\text { Leland } \text { et al. } \\
\mathbf{( 2 0 1 0 )}[\mathbf{2 0}]\end{array}$ & $\begin{array}{c}\text { Schulz } \text { et al. } \\
(\mathbf{2 0 1 0})[\mathbf{2 1}]\end{array}$ & This paper \\
\hline Sensor type & AMR & Piezoelectric & Rogowski coil & Faraday induction \\
Sensor size & $15.6 \times 17.8 \times 2 \mathrm{~mm}$ & $1 \times 0.2 \mathrm{~mm}$ & $2.5 \times 4.5 \times 0.45 \mathrm{~mm}$ & $10 \times 5 \mathrm{~mm}$ \\
Circuit gain & $80 \mathrm{~dB}$ & $40 \mathrm{~dB}$ & $100 \mathrm{~dB}$ & $66.9 \mathrm{~dB}$ \\
$\begin{array}{c}\text { Sensitivity* } \\
\text { DC capable }\end{array}$ & $80 \mu \mathrm{V} / \mathrm{A}$ & $0.87 \mathrm{mV} / \mathrm{A} @ 60 \mathrm{~Hz}$ & $116 \mathrm{nV} / \mathrm{A}-\mathrm{Hz}$ & $21 \mu \mathrm{V} / \mathrm{A} @ 60 \mathrm{~Hz}$ \\
$\begin{array}{c}\text { Susceptible to DC magnetic } \\
\text { field }\end{array}$ & Yes & No & No & No \\
$\begin{array}{c}\text { Household two-wires } \\
\text { application }\end{array}$ & Yes & Not available & No & No \\
Manufacturing process & Complicated & Complicated & Simple & Yes \\
\hline \hline
\end{tabular}

*The results derived from the amplified data.

coupling and the feasibility of the flexible inductive coil tag for current sensing. Fig. 8 shows the measured voltage induced in the coil versus the input current in the cord. The data has been converted into the intrinsic signals without amplification. For a 30-turns inductive coil tag, a linear relation of the induced voltage to the input current with a sensitivity of $18 \mu \mathrm{V} / \mathrm{A}$ and $21 \mu \mathrm{V} / \mathrm{A}$ can be accomplished respectively, while the tag is used for detecting the 50 and $60 \mathrm{~Hz}$ electric current inputs in a $2 \mathrm{C}$ power cord in the ampere regime. However, instead of $22.2 \mu \mathrm{V} / \mathrm{A}$ and $26.6 \mu \mathrm{V} / \mathrm{A}$ calculated by the model, the corresponding measurements, $18 \mu \mathrm{V} / \mathrm{A}$ and $21 \mu \mathrm{V} / \mathrm{A}$, are not as good as expected. The coupling efficiency is only $80 \%$ of the theoretical value. It could be attributed to poor attachment of the sensor tag to the power cord. Fig. 8 also shows the measurement versus the calculated values with different gap distances. While the gap is changed from the idea value, i.e. $1.8 \mathrm{~mm}$, to $2.3 \mathrm{~mm}$, the calculated data can well fit with the experimental one.

The sensitivity, in fact, decreases with the gap, which results in lower magnetic flux coupling. Although the flexible inductive coil tag is aimed to take the advantage of a good proximity effect on the current sensing, the connecting wires soldered to the tag would make the coil structure difficult in closely attaching to the power cord by manual control. Owing to the process characteristic of the flexible tag which is fully compatible with the previously developed SU-8 flexible technology, it is our belief that the sensitivity reduction problem can be further resolved by integrating the coil tag with a CMOS readout circuit chip which can provide a wireless data transmission function as a result of easy implementation and good proximity.

In addition, Eq. (3) indicates that the sensitivity can be substantially enhanced using the coil design with more turns via the reduction of line width and spacing. Nevertheless, the line width and spacing are limited to the manufacturing process. For a conventional roll-to-roll technology [15], [16], similar sensor tag can be realized using a screen-printing process on a flexible substrate, i.e. copper-clad polyimide or
PET film, to fabricate the inductive coil sensor with bonding pads to connect discrete sensing circuit components. The screen-printing process can merely produce the line width and via-filling hole of the coil with the size of several tens of micrometers [16] which would limit the feasibility of sensor sensitivity enhancement by increasing coil turns. In contrast, the inductive coil fabricated by the proposed manufacturing process using advance IC-photolithography technology on a uniformly flexible SU-8 film before the device release from the silicon substrate can have much smaller line width and spacing, i.e. less than $1 \mu \mathrm{m}$, which will make a coil design with more turns in the same tag area. For example, for the coil design of $5 \mu \mathrm{m} / 5 \mu \mathrm{m}$ line width/spacing, Eq. (3) shows $\sim 0.24 \mathrm{mV}$ induced voltage can be achieved with a 220-turn coil design in the same $0.5 \times 1 \mathrm{~cm}^{2}$ area. About an order of magnitude enhancement can be achieved in the induced voltage and a higher sensitivity can be expected. On the other hand, although the induced voltage will be saturated with the coil turns which is caused by less magnetic flux contribution from the inner coils closely to the central region, it can be resolved by incorporating a ferromagnetic core instead of increasing coil numbers. The incorporation of a ferromagnetic core, however, still needs an antimagnetic packaging design to prevent the inevitable influence from the DC component of magnetic field. Related technology development is underway.

Although the gap variation can affect the measurement accuracy, it can be resolved by appropriate calibration. According to Eq. (3), the induced voltage is a function of the gap spacing $(g)$ between the coil and the power cord. As long as the other geometry factors of the coil are fixed and known, $g$ can be easily calculated from the measured voltage value. In other words, if there are two identical coils which are designed and stacked at the same planar location but with different gaps to the power cord, these gap values can then be calculated from the induced voltages when the gap difference between the two identical coils are known [17], [18]. In fact, the gap difference between two identical coils can be well realized and controlled for the tag fabrication also by taking the advantage of advance 
IC-photolithography technology. Similar calibration scheme can be applied for the sensor with planar location variance by designing two identical coils on the same plane but with a fixed distance. Since the calibration can be done using Eq. (3) and a microprocessor unit, good device accuracy can be realized with further development in custom IC design.

The flexible sensor tag is compared with the state-of-theart current sensors as shown in Table I [19]-[21]. Basically, all of these sensors require amplified circuits, but the circuit specification of the presented one is not the most stringent in comparison with the others. Meanwhile, the coil size is much smaller than the wavelength of the background $60 \mathrm{~Hz}$ EM wave $(\sim 5000 \mathrm{~km})$ as a result of low antenna gain. The signal coupled from the environment EM field would be extremely small. In addition, the voltage induction of the presented sensor is irrelevant to any material properties. The sensor can be important because it is not sensitive to DC component of magnetic field and environmental temperature in contrary to other magnetic sensors, e.g. AMR and Hall sensor. Thus, good proximity and reliability indicate that the inductively sensing mechanism is feasible and the sensor can prevail in the applications of household electricity monitoring systems.

\section{CONCLUSION}

A flexible inductive coil tag is demonstrated with the potential for the application to low-cost, reliable and pervasive DR electricity monitoring systems for residential power management. A physical model has been derived and verified for the coil design. According to the model, the device sensitivity can be improved and determined immediately by reducing the metal width and increasing the coil number simultaneously.

\section{ACKNOWLEDGMENT}

The authors would like to thank the Nano Facility Center in National Chiao Tung University, Hsinchu, Taiwan, for the support of fabrication facility and the National Center for High-Performance Computing, Hsinchu, Taiwan, for the support of electromagnetic simulator.

\section{REFERENCES}

[1] E. A. Arens, D. Auslander, D. Culler, C. Federspiel, C. Huizenga, J. Rabaey, P. Wright, and D. White. (2005, Nov.). Demand Response Enabling Technology Development, Phase I Report. Center Built Environment, UC Berkeley, Berkeley [Online]. Available: http://www. escholarship.org/uc/item/0971h43j

[2] C. Xiao, "An overview of integratable current sensor technologies," in Proc. 38th IEEE Annu. Meet. Ind. Appl., vol. 2. Salt Lake City, UT, Oct. 2003, pp. 1251-1258.

[3] P. Ripka, "Electric current sensors: A review," Meas. Sci. Technol., vol. 21, no. 11, pp. 112001-1-112001-23, Sep. 2010.

[4] S. Ziegler, R. C. Woodward, H. H. Iu, and L. J. Borle, "Current sensing techniques: A review," IEEE Sensors J., vol. 9, no. 4, pp. 354-376, Apr. 2009.

[5] P. Ripka, "Current sensors using magnetic materials," J. Optoelectron. Adv. Mater., vol. 6, pp. 587-592, Jun. 2004.

[6] D. A. Ward and J. L. T. Exon, "Using Rogowski coils for transient current measurements," Eng. Sci. Educ. J., vol. 2, no. 3, pp. 105-113, Jun. 1993.

[7] W. F. Ray and C. R. Hewson, "High performance Rogowski current transducers," in Proc. IEEE Annu. Meet. Ind. Appl., vol. 5. Rome, Italy, Oct. 2000, pp. 3083-3090.
[8] T. Waeckerlé, H. Fraisse, Q. Furnemont, and F. Bloch, "Upgrade Fe$50 \% \mathrm{Ni}$ alloys for open-loop DC current sensor: Design and alloypotential characteristics," J. Magn. Magn. Mater, vol. 304, no. 2, pp. e850-e852, Sep. 2006.

[9] H. G. Kim, G. B. Kang, and D. J. Nam, "Coreless hall current sensor for automotive inverters decoupling cross-coupled field," J. Power Electron., vol. 9, no. 1, pp. 68-73, Jan. 2009.

[10] O. Zorlu, P. Kejik, and W. Teppan, "A closed core microfluxgate sensor with cascaded planar FeNi rings," Sensor Actuat. A: Phys., vol. 162, no. 2, pp. 241-247, Aug. 2010.

[11] E. S. Leland, P. K. Wright, and R. M. White, "Design of a MEMS passive, proximity-based AC electric current sensor for residential and commercial loads," in Proc. PowerMEMS, Freiburg, Germany, Nov. 2007, pp. 77-80.

[12] E. S. Leland, R. M. White, and P. K. Wright, "Design and fabrication of a MEMS AC electric current sensor," in Proc. CIMTEC, Jun. 2008, pp. $1-6$.

[13] S. Tumanski, "Induction coil sensors-a review," Meas. Sci. Technol., vol. 18, no. 3, pp. R31-R46, Jan. 2007.

[14] T. Y. Chao and Y. T. Cheng, "Wafer-level chip scale flexible wireless microsystem fabrication," in Proc. IEEE MEMS, Cancun, Mexico, Jan. 2011, pp. 344-347.

[15] C. W. P. Shi, X. Shan, G. Tarapata, R. Jachhowicz, C. W. Lu, and H. T. Hui, "Fabrication of wireless sensor platform on transparent flexible film using screen printing and via interconnect," in Proc. DTIP, Seville, Spain, May 2010, pp. 209-214.

[16] D. Numakura, "Advanced screen printing "practical approaches for printable \& flexible electronics," in Proc. IMPACT, Taipei, Taiwan, Oct. 2008, pp. 205-208.

[17] G. Y. Tian, Z. X. Zhao, and R. W. Baines, "The research of inhomogeneity in eddy current sensors," Sensors Actuat. A: Phys., vol. 69, no. 2, pp. 148-151, Aug. 1998.

[18] X. Chen and T. Ding, "Flexible eddy current sensor array for proximity sensing," Sensors Actuat. A: Phys., vol. 135, no. 1, pp. 126-130, Mar. 2007.

[19] A. M. Pernia, J. M. Lopera, M. J. Prieto, F. Nuno, and S. Ollero, "Characteristics and design of a current sensor using multilayer $\mathrm{Co} / \mathrm{Ni}$ structures," in Proc. APEC, vol. 1. Anaheim, CA, Feb. 1998, pp. 414419.

[20] E. S. Leland, C. T. Sherman, P. Minor, R. M. White, and P. K. Wright, "A new MEMS sensor for AC electric current," in Proc. IEEE Sensors, Waikoloa, HI, Nov. 2010, pp. 1177-1182.

[21] C. A. Schulz, S. Duchesne, D. Roger, and J. Vincent, "Short circuit current measurements between transformer sheets," IEEE Trans. Magn., vol. 46, no. 2, pp. 536-539, Feb. 2010.

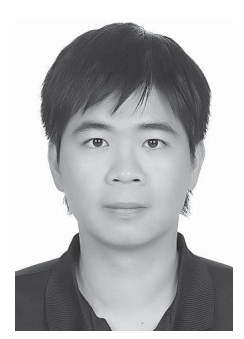

Yung-Chang Chen was born in Taiwan. He received the B.S. degree in electrical engineering from National Taiwan Ocean University, Keelung, Taiwan, and the M.S. degree in electronics engineering from National Chiao Tung University, Hsinchu, Taiwan, in 2006 and 2008, respectively. $\mathrm{He}$ is currently pursuing the Ph.D. degree with the Department of Electronics Engineering, Microsystems Integration Laboratory and the Institute of Electronics, National Chiao Tung University.

His current research interests include microelectromechanical acoustic devices for hearing aid applications and electric sensors for household two-wire application.

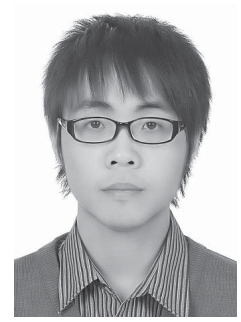

Sung-Chu Yu was born in Taiwan. He received the B.S. degree in physics from National Chung Cheng University, Chiayi, Taiwan, and the M.S. degree in electronics engineering from National Chiao Tung University, Hsinchu, Taiwan, in 2009 and 2011, respectively. His Masters thesis focused on the development of the current sensor for household two-wire application.

He is currently a Research and Development Engineer with the Advanced Technological Development Logic Department, United Microelectronics Corpo- 


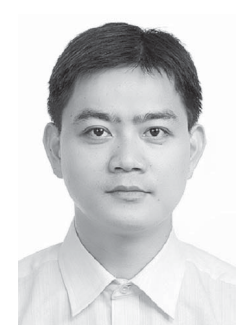

Shih-Hsien Cheng was born in Chiayi, Taiwan, in 1975. He received the B.S. degree in control engineering from Feng Chia University, Taichung, Taiwan, and the M.S. degree in electrical engineering from National Sun Yat-Sen University, Kaohsiung, Taiwan, in 1998 and 2000, respectively. He is currently pursuing the Ph.D. degree with the Institute of Electrical Control Engineering, National Chiao Tung University, Hsinchu, Taiwan.

He is a Research Staff Member with Green Energy and Environment Research Laboratories, Industrial Technology Research Institute, Chutung, Taiwan. His current research interests include power electronics circuit and system designs, neural networks, fuzzy theory, and wireless sensor networks.

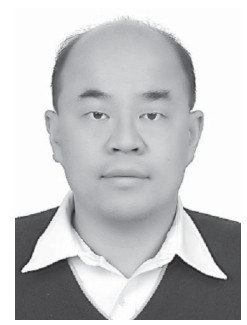

cations.

He was in Army service in Taiwan for two years. He became a Research Staff Member with IBM Thomas J. Watson Research Center, Yorktown Heights, NY, after finishing his Ph.D. in 2000, where he was involved in several system-on-a-package (SoP) projects. In 2002, he joined the Department of Electronics Engineering, National Chiao Tung University (NCTU), Hsinchu, as an Assistant Professor, and was promoted to Professor in 2009. Currently, he serves NCTU as the Director of the Continuing Education Center and Vice-Provost of Academic Affairs. His current research interests include fundamental study of materials for microsystem integration and nano/MEMS applications, SoP, and the design and fabrication of microsensors and microactuators.

Dr. Cheng was a co-recipient of the Best Paper Award in 2006, presented at the 13th IEEE International Conference on Electronics, Circuits, and Systems. He served as a TPC Member of IEEE NEMS in 2011, IEEE ISMM in 2012, and APCOT in 2012. He is a member of Institute of Physics and Phi Tau Phi. 